\title{
PECULIARITIES OF INTRAUTERINE PATHOLOGICAL PROCESSES IN WOMEN OF THE POSTMENOPAUSEAL PERIOD
}

\author{
Tetiana Polishchuk \\ Department of Obstetrics, Gynecology and Perinatology ${ }^{l}$ \\ Serhii Vdovichenko \\ Department of Obstetrics, Gynecology and Perinatology ${ }^{l}$ \\ Oleksandra Lubkovska \\ Department of Obstetrics, Gynecology and Perinatology ${ }^{l}$ \\ Dmytro Ledin \\ Department of Obstetrics, Gynecology and Perinatology ${ }^{l}$ \\ ${ }^{1}$ Ukrainian State Institute of Reproductology \\ Shupyk National Healthcare University of Ukraine \\ 9 Dorohozhytska str., Kyiv, Ukraine, 04112
}

\begin{abstract}
Aim of the research is studying the pecularities of the endometrium pathological processes in postmenopausal women on the basis of the hysteroscopy data evaluation, as well as conduction of histopathological and immunochemical studies.

Materials and methods. To study the pecularities of intrauterine pathological processes, 100 postmenopausal women were selected and studied. All women underwent hysteroscopy with separate diagnostic scraping of the uterine cavity. Also, 10 samples of glandular-fibrous endometrial polyps (GFEP) in women of reproductive age and 9 samples of such pathology in the postmenopausal period were studied by immunohistochemical method, using 6 primary specific monoclonal antibodies.

Results. The results showed that in 64 (64\%) postmenopausal women pathological changes of the endometrium were presented with GFEP, and in 2 patients ( $2 \%$ ) endometrial adenocarcinoma was found. It was also determined that the nuclei of epithelial and stromal cells of GFEP in postmenopausal women are characterized by lower expression of estrogen and progesterone receptors, compared with women with such formations in the reproductive age. In addition, it was found that in postmenopausal women the expression of the apoptosis inhibitor bcl-2 and aromatase P450 in the epithelial and stromal cells of GFEP was more expressed, and the expression of the Bax antigen, on the contrary, was significantly lower than in women of reproductive age.

Conclusions. Fundamental molecular-biological differences of GFEP in postmenopausal women compared with women of reproductive age were revealed. It was found that in postmenopausal women there is a significantly lower dependence of such polyps on the effects of estrogen and progesterone. In addition, the data indicate an increased risk of neoplastic transformation in such women.

Keywords: postmenopause, pathological process of the endometrium, glandular-fibrous endometrial polyp, endometrial adenocarcinoma.
\end{abstract}

\section{Introduction}

Problems of the postmenopausal period, in particular prevention, diagnosis and treatment of endometrial pathology in elderly patients, have become especially relevant in gynecology. Elderly women are at risk for a variety of intrauterine pathology on the background of involutive changes in the reproductive system. Pathological processes of the endometrium (PPE) in such women are characterized by a high risk of malignancy and problems associated with dysfunctional uterine bleeding and anemia $[1,2]$. Thus, according to various studies, about $50 \%$ of cases of endometrial cancer in postmenopause are the result of malignancy of endometrial hyperplastic processes. The frequency and timing of malignancy vary widely and largely depend on the severity of hyperproliferation in the endometrium, the presence of hormonal and metabolic disorders. It is also known that the risk of PPE malignancy is 2.5 times higher in women whose menstrual function ended after 50 years, compared with women whose menopause occurred before 50 years $[3,4]$. 
In addition, it was found that hyper- and neoplastic processes in the uterine mucosa develop against the background of a progressive decrease in the ability of cells to apoptosis and increase the intensity of neoangiogenesis. Therefore, much attention is paid to proteins of the bcl-2 family, which can promote or inhibit apoptosis by synthesizing antiapoptotic (namely bcl-2, bcl-Xl) or proapoptotic (for example, Bax, Вак, Bad, Bid, bcl-X) proteins and others [5, 6].

In the majority of postmenopausal women, PPE is asymptomatic, which does not exclude the presence of a pathological process in the involutive endometrium and which are detected only during ultrasound diagnostics [7, 8]. Therefore, the diagnosis of PPE in the postmenopausal period, in particular on the basis of histopathological and immunochemical studies, remain relevant in modern medicine.

The aim is to study the features of pathological processes of the endometrium in postmenopausal women on the basis of evaluation of hysteroscopy data, as well as conduction of the histopathological and immunochemical studies.

\section{Materials and methods}

The study was conducted from 2017 to 2019 on the basis of the Department of Obstetrics, Gynecology and Perinatology of Shupyk National Healthcare University of Ukraine, on the basis of the Municipal Non-Profit Enterprise «Vinnytsia Central District Clinical Hospital», as well as on the pathomorphology department of State Institution «Institute Of Pediatrics, Obstetrics And Gynecology» Named After Academician O.M. Lukyanova of the National Academy Of Medical Sciences Of Ukraine.

In order to analyze the features of intrauterine pathological processes in the postmenopausal period, 100 women were selected. The age of patients ranged from 60 to 85 years (mean age $68.5 \pm 5.7$ years). The period when menopause occurred ranged from 42 to 57 years. The average menopause occurred at the age of $47.8 \pm 5.7$.

The study was conducted in accordance with the Helsinki Declaration according to the conclusion of the Ethics Commission of Shupyk National Healthcare University of Ukraine (Protocol No. 3 from 07.02 .2017 ). Informed consent to participate in the study was obtained from all women.

From the anamnesis it was established that 10 women (10\%) did not become pregnant, 40 women (40\%) had one childbirth, 35 patients (35\%) had two and 15 women (15\%) had three or more births.

From the gynecological anamnesis it was found that 20 women $(20 \%)$ had uterine fibroids, 30 patients $(30 \%)$ had endometrial pathology, 25 women $(25 \%)$ had cervical pathology and pelvic inflammatory disease (PID), and 10 women (10\%) - ovarian cysts.

All patients admitted to the gynecology department with suspected PPE were routinely examined. At the outpatient stage, they underwent a complete clinical and laboratory examination, including complaint analysis, detailed history taking, gynecological examination, blood type, rhesus factor, RW, HIV, HBsAg, HCV, clinical blood test, general urine test, biochemical blood test, coagulogram, smears on flora and urogenital infections, smears on oncocytology of the cervix, ECG, chest radiography.

In addition to general clinical, all patients underwent gynecological examination, including examination of the external genitalia, cervix in mirrors with simple and advanced colposcopy, bimanual examination to determine the position, size, density, motility of the uterus and appendages, depth and nature of discharge.

Hysteroscopy with separate-diagnostic scraping of the cervical canal and uterine cavity was performed in the gynecological department in a small operating room under general short-term intravenous anesthesia. When performing diagnostic and therapeutic hysteroscopy operating and contact hysteroscope «Olympus $4 \mathrm{~mm}$ » was used. The outer diameter of the hysteroscopes was $8 \mathrm{~mm}$, the corresponding telescopes had a diameter of $4 \mathrm{~mm}$. Turusol, sterile saline and $5 \%$ glucose solution were used as liquid media. Separate diagnostic scraping of the cervical canal and uterine cavity was performed under aseptic conditions after treatment of the external genitalia and vaginal mucosa with $1 \%$ solution of iodopyrone, the cervix was grasped with ball forceps. Dilation of the cervical canal was performed using Gegar dilators up to No. 9. The cervical canal 
was scraped with a curette No. 1, then the uterine cavity was scraped with a No. 2, 4, 6 curette. Scrapers were placed in separate plastic containers, and then sent to the pathology department. The material for optical examination was fixed in $10 \%$ neutral formalin, carried out through alcohols of increasing concentration $\left(50-96^{\circ}\right)$, poured into histomyx, and then on a rotary microtome Sakura made sections 5-6 $\mu \mathrm{m}$ thick, which were waxed and stained with hematoxylin and eosin .

Immunohistochemical study of 10 samples of GFEP in women of reproductive age $(10 \mathrm{ob}-$ servations) and menopause ( 9 patients aged 64-81 years), using 6 primary specific monoclonal antibodies, namely: to estrogen receptors (rabbit monoclonal antibody, IgG, C-term, Spring Bioscience, USA); to progesterone receptors (rabbit monoclonal antibody, IgG, C-term, Spring Bioscience, USA); to Ki-67 protein (mouse monoclonal antibody, IgG1, Leica, UK); to the apoptosis inhibitor bcl-2 (Bcl-2, mouse monoclonal antibody, IgGl, Cell Marque, USA); to the apoptosis inducer Bax (Bax-antibody, rabbit monoclonal antibody, IgG, GeneTex, USA); to cytochrome 450 aromatase (cytochrome 450 - rabbit monoclonal antibody, IgG, GeneTex, USA) was conducted. The results were evaluated by calculating the expression coefficients of an antigen.

The 2007 Microsoft Office package, in particular Excel 2007, was used for the statistical analysis of the obtained results. Evaluation of the statistical significance of the obtained data was performed using Student's t-test. The differences were considered statistically significant at $p<0.05$.

\section{Results of the research}

The obtained results showed that out of 100 postmenopausal women with suspected PPE during gynecological examination, 11 women (11\%) had atresia of the external pharynx, another ten $(10 \%)$ had atresia of the internal pharynx detected during uterine probing during surgery.

All women underwent separate diagnostic scraping of the cervical canal and uterine cavity with prior hysteroscopy. Thus, the hysteroscopic picture of the uterine cavity in such women was characterized by the presence of polyps of different size and location, inhomogeneous local thickening of the endometrium, the presence of submucosal or interstitial submucosal fibroids, synechiae in the uterine cavity, signs of endometrial atrophy and chronic endometritis.

14 patients $(14 \%)$ underwent surgical hysteroscopy using endoscopic forceps and scissors (removal of small polyps from the tube angles), 11 women (11\%) underwent hysteroresectoscopy (removal of fibroids and polyps on a broad basis, which could not be removed by curettage), as well as 6 patients $(6 \%)$ endometrial ablation was conducted.

Pathohistological examination of $70(70 \%)$ patients revealed pathological changes in the endometrium. In addition, in 25 (25\%) women only atrophy of the endometrium, and in 5 (5\%) submucosal nodes of uterine fibroids (Table 1) were histologically determined.

Table 1

The structure of PPE in postmenopausal women

\begin{tabular}{lcc}
\hline \multicolumn{1}{c}{ Nosological unit } & Abs. & \% \\
\hline No pathological changes were detected, atrophic endometrium & 25 & $25 \%$ \\
Glandular-fibrous endometrial polyp & 64 & $64 \%$ \\
Chronic endometritis & 4 & $4 \%$ \\
Endometrial adenocarcinoma & 2 & $2 \%$ \\
Submucosal nodes & 5 & $5 \%$ \\
Total & 100 & $100 \%$
\end{tabular}

Thus, most often PPE in postmenopausal women was characterized by glandular-fibrous polyps (64\%, namely in 64 patients). At the same time, there were a high percentage of atrophic processes of the endometrium, which was due to age-related changes, as well as a high frequency of malignant endometrial tumors.

In order to further analyze of GFEP, as the most important pathology in the structure of PPE, the examined women underwent immunohistochemical study. For this purpose, 6 primary 
specific monoclonal antibodies to estrogen receptors, progesterone, Ki-67 protein, bcl-2 apoptosis inhibitor and apoptosis inducer Bax were used. Their expression by epithelial and stromal cells of GFEP was compared between women in the postmenopausal and reproductive periods.

Analysis of the obtained data showed that in postmenopausal women the expression of estrogen and progesterone receptors by the nuclei of epithelial and stromal cells of GFEP was statistically significantly lower compared to women of reproductive age (Table 2).

Table 2

Expression coefficients $(M \pm m)$ of estrogen and progesterone receptors by the nuclei of epithelial and stromal cells of GFEP

\begin{tabular}{lcccc}
\hline \multirow{2}{*}{ Indicator } & \multicolumn{2}{c}{ Women of reproductive age $(\boldsymbol{n}=\mathbf{1 0})$} & \multicolumn{2}{c}{ Postmenopausal women $(\boldsymbol{n}=\mathbf{9})$} \\
\cline { 2 - 5 } & Epithelium & Stroma & Epithelium & Stroma \\
\hline Estrogen receptors expression & $2.04 \pm 0.06$ & $1.53 \pm 0.09$ & $1.03 \pm 0.07^{*}$ & $0.61 \pm 0.05^{*}$ \\
Progesterone receptors expression & $0.87 \pm 0.09$ & $1.12 \pm 0.05$ & $0.42 \pm 0.04^{*}$ & $0.53 \pm 0.06^{*}$
\end{tabular}

Note: $*-p<0.01$ compared with GFEP indices in women of reproductive age

In the study of Ki-67 expression, it was found that for all studied GFEP, regardless of age and period of life of women, the expression of the marker of proliferation in the glandular epithelium was similar, namely proliferative activity was significantly expressed in both observation groups. However, in contrast to GFEP in the reproductive period, in such polyps in postmenopausal women, the expression of Ki-67 in the nuclei of stromal cells was more expressed (Table 3).

Table 3

Coefficient of expression $(M \pm m)$ of Ki-67 by the nuclei of epithelial and stromal cells of GFEP

\begin{tabular}{ccccc}
\hline \multirow{2}{*}{ Indicator } & \multicolumn{2}{c}{ Women of reproductive age $(\boldsymbol{n}=\mathbf{1 0})$} & \multicolumn{2}{c}{ Postmenopausal women $(\boldsymbol{n}=\mathbf{9})$} \\
\cline { 2 - 5 } & Epithelium & Stroma & Epithelium & Stroma \\
\hline Ki-67 expression & $0.55 \pm 0.13$ & $0.39 \pm 0.11$ & $0.48 \pm 0.04$ & $0.65 \pm 0.01^{*}$
\end{tabular}

Note: ${ }^{*}-p<0.05$ compared with GFEP indices in women of reproductive age

The level of such an indicator as a bcl-2 apoptosis inhibitor was also determined. Thus, in comparison with GFEP in women of reproductive age, in polyps of postmenopausal women, its expression in epithelial and stromal cells was more expressed (Table 4).

Table 4

Coefficients of expression $(M \pm m)$ of apoptosis inhibitor bcl-2 by epithelial and stromal cells of GFEP

\begin{tabular}{ccccc}
\hline \multirow{2}{*}{ Indicator } & \multicolumn{2}{c}{ Women of reproductive age $(\boldsymbol{n}=\mathbf{1 0})$} & \multicolumn{2}{c}{ Postmenopausal women $(\boldsymbol{n}=\mathbf{9})$} \\
\cline { 2 - 5 } & Epithelium & Stroma & Epithelium & Stroma \\
\hline bcl-2 expression & $0.22 \pm 0.02$ & $0.11 \pm 0.01$ & $0.49 \pm 0.04^{*}$ & $0.38 \pm 0.03^{*}$
\end{tabular}

Note: $*-p<0.01$ compared with GFEP indices in women of reproductive age

With regard to the expression of the inducer of apoptosis, the Bax antigen, in polyps in postmenopausal women, its expression in epithelial and stromal cells was much weaker (Table 5). Thus, the processes of apoptosis in GFEP in postmenopausal women are significantly suppressed, in contrast to GFEP in women of reproductive age. As a result, despite the absence of statistically significant differences in the proliferative activity of the epithelium and its increase only in the stroma, the suppression of apoptosis in GFEP in menopause indicates an increased risk of neoplastic transformation. 
Table 5

Expression coefficients $(M \pm m)$ of the apoptosis inducer (Bax) by epithelial and stromal cells of GFEP

\begin{tabular}{ccccc}
\hline \multirow{2}{*}{\begin{tabular}{c} 
Indicator \\
\cline { 2 - 5 }
\end{tabular}} & \multicolumn{2}{c}{ Women of reproductive age $(\boldsymbol{n}=\mathbf{1 0})$} & \multicolumn{2}{c}{ Postmenopausal women $(\boldsymbol{n}=\mathbf{9})$} \\
\cline { 2 - 5 } & Epithelium & Stroma & Epithelium & Stroma \\
\hline Bax expression & $0.68 \pm 0.05$ & $0.62 \pm 0.05$ & $0.38 \pm 0.04^{*}$ & $0.33 \pm 0.03^{*}$
\end{tabular}

Note: * $-p<0.001$ compared with GFEP indices in women of reproductive age

In addition to the above indicators, the expression of aromatase $\mathrm{P} 450$ was also evaluated. Thus, in comparison with GFEP in the reproductive period, with polyps in postmenopausal women in varying degrees, from minimal to moderate, there was a expressed expression, but mainly in the epithelium of the glands (Table 6). This is a fundamental difference between GFEP in postmenopause, which indicates the local synthesis of estrogen in such polyps.

Table 6

Coefficient of expression $(M \pm m)$ of aromatase P450 by epithelial and stromal cells of GFEP

\begin{tabular}{ccccc}
\hline \multirow{2}{*}{ Indicator } & \multicolumn{2}{c}{ Women of reproductive age $(\boldsymbol{n}=\mathbf{1 0})$} & \multicolumn{2}{c}{ Postmenopausal women $(\boldsymbol{n}=\mathbf{9})$} \\
\cline { 2 - 5 } & Epithelium & Stroma & Epithelium & Stroma \\
\hline P450 expression & - & - & $0.12 \pm 0.02 *$ & $0.09 \pm 0.01^{*}$
\end{tabular}

Note: ${ }^{*}-p<0.01$ compared with GFEP indices in women of reproductive age

Thus, the results obtained by immunohistochemical study showed that postmenopausal women with GFEP, in contrast to such pathology in the reproductive period, are characterized by less influence of estrogen and progesterone on the development of such polyps. The study also found an increased risk of neoplastic transformation in postmenopausal women.

\section{Discussion}

The hormonal background of the female body changes throughout life - from early reproductive age to postmenopause. According to the available data, the changes that occur during premenopause, menopause and postmenopause can cause various pathological processes of the endometrium. Such processes require considerable attention and timely diagnosis, as they can lead to the development of cancer. The cause of such disorders is usually called hormonal imbalance, in particular the development of hyperestrogenism, as well as a progressive decrease in the ability of cells to apoptosis and increase the intensity of neoangiogenesis $[9,10]$.

In this context, it should be noted that the pathology of the endometrium is one of the diseases that is more common in postmenopausal women. Thus, according to a number of studies, in particular [11, 12], such pathology in $90 \%$ of cases occurs in women over 50 years of age. In addition, a number of researchers indicate that PPE in such women are mainly represented by endometrial polyps, submucosal uterine fibroids, chronic endometritis, synechiae. Also, compared with women of reproductive age, endometrial adenocarcinoma is more common [13, 14].

As a result of the histopathological examination the following structure of PPE was revealed: glandular-fibrous endometrial polyp - $64 \%$, or in 64 women; atrophic endometrium $-25 \%$, or in 25 patients; submucosal nodes $-5 \%$, or in 5 patients; chronic endometritis $-4 \%$, or in 4 women; endometrial adenocarcinoma $-2 \%$, or in 2 women.

In order to study the peculiarities of the development of such processes and their comparison in different periods, namely in postmenopause and reproductive age, to increase the effectiveness of their early diagnosis, an in-depth immunohistochemical study of GFEP, as a disorder occupying the largest share, were conducted.

Given that the causes of PPE may be absolute and relative hyperestrogenism with corresponding changes in progesterone levels, and, at the same time, the lack of unambiguous data on 
the role of these processes in the postmenopausal period in the development of GFEP, comparison of the expression of estrogen receptors and progesterone stromal cells in women of postmenomasal and reproductive age was conducted.

Analysis of the data showed that the expression of estrogen receptors was statistically significantly lower in postmenopausal women compared with women of reproductive age. A similar pattern was observed with respect to the expression of progesterone receptors, namely its significantly lower expression by the nuclei of epithelial and stromal cells of GFEP in the postmenopausal period, compared with women of reproductive age.

The development of PPE also depends on the characteristics of the proliferative activity of the endometrium, which is facilitated by proliferation factors, in particular Ki-67, which are necessary for the replication of genomic DNA. At the same time, in the literature there are debatable data on changes in the proliferation factor of Ki-67 in hyperplasia compared with normal endometrium, in particular on the differences between postmenopause and reproductive age $[15,16]$. The results of the study showed that its proliferative activity was significantly expressed in both groups. At the same time, in the nuclei of GFEP stromal cells in postmenopausal women, the expression of Ki-67 was more significant, in contrast to GFEP in women of reproductive age.

In addition to proliferative factors, the development of PPE is also influenced by antiapoptotic, especially bcl-2, and proapoptotic, in particular Bax, proteins that act as inhibitors or inducers of apoptosis. In this context, there is a hypothesis that polyps are not essentially mitotically hyperactive, but develop due to a defect in the mechanisms of natural control of apoptosis, which leads to prolonged cell life $[17,18]$.

The results of the study showed that in comparison with GFEP patients of reproductive age, in polyps of postmenopausal women, the expression of bcl-2 in epithelial and stromal cells was significantly higher. Another picture was observed for the Bax antigen, which was characterized by significantly lower expression in the epithelial and stromal cells of GFEP in postmenopausal women than in polyps in women of reproductive age [19].

The local increase in the estrogen concentration, which enhances cell proliferation, indicates the expression of aromatase cytochrome 450. Under conditions of apoptosis suppression, this increases the risk of neoplastic transformation [20,21]. The obtained data showed the presence of such expression in GFEP of postmenopausal women, in contrast to such formations in women of reproductive age.

Study limitations. The study did not include women who had acute infectious diseases and women with severe extragenital diseases who were contraindicated surgeries under anesthesia.

Prospects for further research. Improving the effectiveness of early diagnosis of PPE in postmenopausal women to reduce the risk of their malignant transformation.

\section{Conclusions}

The results obtained during the study made it possible to identify the anatomical features of the uterus in the postmenopausal period, in particular partial or complete atresia of the cervical canal.

It was also found that the most common pathology of the endometrium in postmenopausal women are glandular-fibrous endometrial polyps (64\%, namely in 64 women), and there is a high risk of endometrial adenocarcinoma ( $2 \%$, namely in 2 women).

The obtained data of immunohistochemical study showed that in GFEP patients in postmenopausal period there was a statistically significant lower expression of both estrogen receptors and progesterone. At the same time, the expression of $\mathrm{Ki}-67$, which is responsible for proliferative activity, was significantly expressed in both women of reproductive age and postmenopausal women. It was also found that the expression of the proliferation marker Ki-67 in the nuclei of stromal cells was more expressed in the postmenopausal period than in the reproductive age.

In addition to the mentioned above, there was an increase in the expression of the apoptosis inhibitor bcl-2 along with a decrease in the expression of the inducer of apoptosis Bax in the epithelial and stromal cells of polyps in postmenopausal women compared with reproductive age. The results indicate that the processes of apoptosis in GFEP in postmenopausal women are significantly suppressed, compared with GFEP in women of reproductive age. Cytochrome 450 aromatase 
expression was also found to be higher than that of women of reproductive age, indicating the likelihood of a local increase in estrogen levels, which in turn will increase cell proliferation. All this together with the suppression of apoptosis may increase the risk of neoplastic transformation.

\section{Conflict of interest}

The authors declare that they have no conflicts of interest.

\section{Financing}

None.

\section{References}

[1] Bueloni-Dias, F. N., Spadoto-Dias, D., Delmanto, L. R. M. G., Nahas-Neto, J., Nahas, E. A. P. (2016). Metabolic syndrome as a predictor of endometrial polyps in postmenopausal women. Menopause, 23 (7), 759-764. doi: http://doi.org/10.1097/ gme.0000000000000616

[2] Morice, P., Leary, A., Creutzberg, C., Abu-Rustum, N., Darai, E. (2016). Endometrial cancer. The Lancet, 387 (10023), 1094-1108. doi: http://doi.org/10.1016/s0140-6736(15)00130-0

[3] Costa-Paiva, L., Godoy, C. E., Antunes, A., Caseiro, J. D., Arthuso, M., Pinto-Neto, A. M. (2011). Risk of malignancy in endometrial polyps in premenopausal and postmenopausal women according to clinicopathologic characteristics. Menopause, 18 (12), 1278-1282. doi: http://doi.org/10.1097/gme.0b013e31821e23a1

[4] Günakan, E., Atak, Z., Albayrak, M., Kurban, Y., Şimşek, G. G. (2018). Endometrial histopathology results and evaluation of endometrial cancer risk in geriatric women. Menopausal Review, 17 (1), 18-21. doi: http://doi.org/10.5114/pm.2018.74898

[5] Pinheiro, A., Antunes, A., Andrade, L., De Brot, L., Pinto-Neto, A. M., Costa-Paiva, L. (2014). Expression of hormone receptors, Bcl-2, Cox-2 and Ki67 in benign endometrial polyps and their association with obesity. Molecular Medicine Reports, 9 (6), 2335-2341. doi: http://doi.org/10.3892/mmr.2014.2125

[6] Banas, T., Pitynski, K., Mikos, M., Cielecka-Kuszyk, J. (2018). Endometrial Polyps and Benign Endometrial Hyperplasia Have Increased Prevalence of DNA Fragmentation Factors 40 and 45 (DFF40 and DFF45) Together With the Antiapoptotic B-Cell Lymphoma (Bcl-2) Protein Compared With Normal Human Endometria. International Journal of Gynecological Pathology, 37 (5), 431-440. doi: http://doi.org/10.1097/pgp.0000000000000442

[7] Adomaitiene, L., Nadisauskiene, R., Nickkho-Amiry, M., Cizauskas, A., Palubinskiene, J., Holland, C., Seif, M. W. (2020). Tumor Suppression in Asymptomatic Postmenopausal Endometrial Polyps. Anticancer Research, 40 (2), 789-794. doi: http://doi.org/ 10.21873/anticanres.14010

[8] Cavkaytar, S., Kokanali, M. K., Ceran, U., Topcu, H. O., Sirvan, L., Doganay, M. (2014). Roles of Sonography and Hysteroscopy in the Detection of Premalignant and Malignant Polyps in Women Presenting with Postmenopausal Bleeding and Thickened Endometrium. Asian Pacific Journal of Cancer Prevention, 15 (13), 5355-5358. doi: http://doi.org/10.7314/apjcp.2014.15.13.5355

[9] Vicennati, V., Garelli, S., Rinaldi, E., Rosetti, S., Zavatta, G., Pagotto, U., Pasquali, R. (2015). Obesity-related proliferative diseases: the interaction between adipose tissue and estrogens in post-menopausal women. Hormone Molecular Biology and Clinical Investigation, 21 (1), 75-87. doi: http://doi.org/10.1515/hmbci-2015-0002

[10] Tsuzuki, Y., Kikuchi, I., Nojima, M., Yoshida, K., Hashizume, A., Tomita, S. (2017). A Case Report: Ovarian Sertoli-Leydig Cell Tumor With Hyperestrogenism and Endometrial Hyperplasia in a Postmenopausal Woman. Japanese Clinical Medicine, 8. doi: http://doi.org/10.1177/1179066017695239

[11] Otify, M., Fuller, J., Ross, J., Shaikh, H., Johns, J. (2014). Endometrial pathology in the postmenopausal woman - an evidence based approach to management. The Obstetrician \& Gynaecologist, 17 (1), 29-38. doi: http://doi.org/10.1111/tog.12150

[12] Siegel, R. L., Fedewa, S. A., Miller, K. D., Goding-Sauer, A., Pinheiro, P. S., Martinez-Tyson, D., Jemal, A. (2015). Cancer statistics for Hispanics/Latinos, 2015. CA: A Cancer Journal for Clinicians, 65 (6), 457-480. doi: http://doi.org/10.3322/caac.21314

[13] Elfayomy, A. K., Habib, F. A., Alkabalawy, M. A. (2012). Role of hysteroscopy in the detection of endometrial pathologies in women presenting with postmenopausal bleeding and thickened endometrium. Archives of Gynecology and Obstetrics, 285 (3), 839-843. doi: http://doi.org/10.1007/s00404-011-2068-6

[14] Genc, M., Genc, B., Sahin, N., Celik, E., Turan, G. A., Gur, E. B., Guclu, S. (2014). Endometrial pathology in postmenopausal women with no bleeding. Climacteric, 18 (2), 241-245. doi: http://doi.org/10.3109/13697137.2014.944152

[15] Dubossarskaya, Z. M., Greek, L. P. (2014). Rationale for treatment of patients with «proliferative syndrome» in gynecology. Zdorove zhenschiny, 3, 145-148.

[16] Shcherbyna, M. O., Viesich, T. L., Shcherbyna, I. M., Taravnekh, D. Sh. (2017). The state of endometrial receptors at hyperplastic processes in perimenopausal women. Mizhnarodnyi medychnyi zhurnal, 23 (2), 37-40. 
[17] Antunes, A., Vassallo, J., Pinheiro, A., Leão, R., Neto, A. M. P., Costa-Paiva, L. (2014). Immunohistochemical expression of estrogen and progesterone receptors in endometrial polyps: A comparison between benign and malignant polyps in postmenopausal patients. Oncology Letters, 7 (6), 1944-1950. doi: http://doi.org/10.3892/ol.2014.2004

[18] Troncon, J. K., Meola, J., Candido-dos-Reis, F. J., Poli-Neto, O. B., Nogueira, A. A., Rosa-e-Silva, J. C. (2017). Analysis of differential genetic expression in endometrial polyps of postmenopausal women. Climacteric, 20 (5), 462-466. doi: http://doi.org/ 10.1080/13697137.2017.1335701

[19] Polishchuk, T. P. (2020). Prophylaxis of relapses for the women of postmenopausal period with of high quality pathology of endometrium. Reproductive health of woman, 3, 24-27. doi: http://doi.org/10.30841/2708-8731.3.2020.215008

[20] Tingting, S. U., Sui, L. (2014). Expression and significance of p63, aromatase P450 and steroidogenic factor-1 in endometrial polyp. Chinese Journal of Obstetrics and Gynecology, 49 (8), 604-608.

[21] Artymuk, N. V., Gulyaeva, L. F., Zotova, O. A. (2012). Metabolism peculiaritirs and estrogenes reception in endometrial hyperplasia and endometriosis. Mat i ditya v Kuzbasse, 1, 8-12.

Received date 16.04.2021

Accepted date 17.05.2021

Published date 25.05.2021
(C) The Author(s) 2021

This is an open access article under the Creative Commons CC BY license

How to cite: Polishchuk, T., Vdovichenko, S., Lubkovska, O., Ledin, D. (2021). Peculiarities of intrauterine pathological processes in women of the postmenopauseal period. EUREKA: Health Sciences, 3, 16-23. doi: http://doi.org/10.21303/2504-5679.2021.001853 